\title{
Neoadjuvant Therapy
}

National Cancer Institute

\section{Source}

National Cancer Institute. Neoadjuvant Therapy. NCI Thesaurus. Code C15665.

The administration of therapeutic agents prior to the primary treatment for the purpose of making the primary treatment more effective. 